\title{
Benzodiazepine resistant alcohol withdrawal: What is the clinician's preferred definition?
}

\author{
Hugo Langlois (1), PharmD, MSc*; Monique Cormier ${ }^{\dagger}$; Eric Villeneuve ${ }^{*}$, PharmD, MSc*; \\ Robert S. Hoffman, $\mathrm{MD}^{\dagger \neq}$; Cristina Longo, $\mathrm{PhD}^{\S}$; Sophie Gosselin $\left(\mathbb{D}, \mathrm{MD}^{\dagger \boldsymbol{\tau}^{* *}}\right.$
}

\section{CLINICIAN'S CAPSULE}

What is known about the topic?

Benzodiazepine-resistant alcohol withdrawal syndrome is not well defined in the literature.

What did this study ask?

We assessed clinicians' personal definition of resistant alcohol withdrawal with a survey-based approach.

What did this study find?

High benzodiazepine dosage $(40 \mathrm{mg} / \mathrm{hr}$ of diazepam equivalents) was considered a determinant for defining resistant alcohol withdrawal.

Why does this study matter to clinicians?

High benzodiazepine usage should prompt clinicians to suspect resistant alcohol withdrawal syndrome and add another molecule such as phenobarbital.

\section{ABSTRACT}

Objectives: Although alcohol withdrawal is common, the recognition of benzodiazepine-resistant alcohol withdrawal is a relatively new concept. To provide a framework for both literature review and future research, we assessed clinicians' personal definition of resistant alcohol withdrawal.

Method: We developed a cross-sectional web-based survey. Administrators from collaborating toxicology and emergency medicine associations deployed the survey directly to their respective memberships. Only physicians, pharmacists, and other clinicians routinely treating alcohol withdrawal were eligible to participate. Respondents selected their preferred definition among the three most common author sources - JB Hack, NJ Benedict, D Hughes - or provided their own. Additional criteria to define resistant alcohol withdrawal were explored.

Results: 384 individuals answered the survey. Respondents were mostly attending physicians $(79 \%)$, in full-time practice $(90 \%)$, in emergency medicine (70\%), and from North America (90\%). The majority (64\%) described resistant alcohol withdrawal as a high benzodiazepine dosage. Seizures (26\%) and persistent tachycardia (16\%) were also main characteristics. The median dose to describe high benzodiazepine dose ( $n=146$ ) was $40 \mathrm{mg}$ per hour of diazepam equivalents (IQR 20-50). Available definitions were ranked equally as the preferred one: Hack (27\%); Benedict (28\%); Hughes (28\%).

Conclusion: Our results did not identify one single preferred definition for resistant alcohol withdrawal even though a high total dose of benzodiazepine is a major component. Hourly requirements of $40 \mathrm{mg}$ of diazepam equivalents or more emerged as a possible threshold. These findings serve as a base to explore consensus guidelines or future research.

\section{RÉSUMÉ}

Objectif: Si le syndrome de sevrage à l'alcool est un trouble fréquent, la reconnaissance du syndrome de sevrage à l'alcool résistant aux benzodiazépines est relativement nouvelle. Afin de fournir un cadre de travail se prêtant aussi bien aux revues de la documentation qu'aux recherches futures, l'équipe a évalué les définitions personnelles que les cliniciens ont du syndrome de sevrage à l'alcool résistant aux benzodiazépines.

Méthode: Nous avons développé une étude transversale par le biais d'un sondage électronique. Les administrateurs des associations participantes en toxicologie et en médecine d'urgence ont mis directement le questionnaire à la disposition de leurs membres respectifs. Seuls les médecins, les pharmaciens et d'autres cliniciens habitués au traitement du syndrome de sevrage à l'alcool pouvaient participer à l'étude. Les répondants devaient choisir, parmi les trois définitions les plus courantes du syndrome de sevrage à l'alcool résistant aux benzodiazépine (Hack, Benedict, Hugues), celle qu'ils préféraient, ou encore donner leur propre définition. D'autres critères visant à définir le syndrome de sevrage à l'alcool résistant ont aussi été examinés. Résultats: Au total, 384 personnes ont répondu au questionnaire. II s'agissait, pour la plupart, de médecins traitants (79\%), pratiquant à temps complet $(90 \%)$, en médecine d'urgence $(70 \%)$ et en Amérique du Nord (90\%). La majorité (64\%) d'entre

From the *Pharmacy Department, McGill University Health Centre, Montreal, QC; †Montreal Medical Toxicology Initiative, Montreal, QC; $\neq$ Division of Medical Toxicology, Ronald O. Perelman Department of Emergency Medicine, New York University School of Medicine, NY; §Department of Family Medicine, McGill University, Montreal, OC; IDepartment of Emergency Medicine, McGill University Health Centre, Montreal, OC; and ${ }^{*}$ Centre antipoison du Québec, Quebec City, QC.

Correspondence to: Hugo Langlois, 1001 Decarie Blvd., Montreal, QC H4A 3J1; Email: hugo.langlois.1@ umontreal.ca

(C) Canadian Association of Emergency Physicians 
eux considérait le syndrome de sevrage à l'alcool comme résistant s'il nécessitait de fortes doses de benzodiazépines. La présence de convulsions (26\%) et une tachycardie persistante (16\%) figuraient aussi parmi les principales caractéristiques. La dose médiane de benzodiazépines considérée comme forte $(n=$ 146) s'élevait à $40 \mathrm{mg}$ en équivalent de diazépam, toutes les heures (intervalle interquartile : 20-50). Les définitions proposées ont recueilli un taux quasi égal de réponse, soit $27 \%$ pour Hack, $28 \%$ pour Benedict et $28 \%$ pour Hugues.

Conclusion: Les résultats de l'étude n'ont pas permis de dégager une seule définition du syndrome de sevrage à l'alcool résistant, mais une dose totale élevée de benzodiazépines est considérée comme un élément important. Par ailleurs, des doses totales de $40 \mathrm{mg}$ ou plus en équivalent de diazépam administrées toutes les heures pourraient marquer un seuil possible. Enfin, les résultats obtenus servent de fondements potentiels à I'élaboration de lignes directrices consensuelles et à de futurs travaux de recherche.

Keywords: Drugs and pharmacology, emergency medicine, resistant alcohol withdrawal, toxicology

\section{INTRODUCTION}

Alcohol withdrawal is a common problem in emergency departments. ${ }^{1,2}$ Although benzodiazepines are the firstline treatment, ${ }^{3,4}$ some withdrawal patients are poorly responsive to benzodiazepines. This entity is often named benzodiazepine-resistant or resistant alcohol withdrawal. Unfortunately, there is no standard definition of resistant alcohol withdrawal. Hack et al. suggested a combination of abnormal vital signs and a cumulative dose of more than $200 \mathrm{mg}$ of diazepam over 3 hours. $^{5}$ However, these criteria were never validated or subjected to expert consensus. Also, the definition of abnormal vital signs was imprecise. All subsequent definitions of resistant alcohol withdrawal seem to be variations on Hack's initial efforts. Among these, one suggests that more than $10 \mathrm{mg}$ of lorazepam in 1 hour or more than $40 \mathrm{mg}$ in 4 hours would define benzodiazepine resistance. ${ }^{6}$ A recent retrospective study defined benzodiazepine resistance based on three different criteria: $200 \mathrm{mg}$ of diazepam in 4 hours; greater than $40 \mathrm{mg}$ of diazepam in 1 hour; or an individual dose of greater than $40 \mathrm{mg}$ of diazepam required to control agitation. ${ }^{7}$ A formalized definition of resistant withdrawal might standardize future research by improving comparability of patients, which ultimately could improve the assessment of interventions on relevant clinical outcomes. The present study assesses clinicians' personal definitions of resistant alcohol withdrawal with a survey-based methodology.

\section{METHODS}

\section{Study design and population}

We designed a 25-item open cross-sectional survey assessing two different aspects of alcohol withdrawal among emergency physicians and toxicologists. The first part assesses clinicians' personal definition of resistant alcohol withdrawal. The questionnaire was imported on the SimpleSurvey platform, a web-based online survey tool. It was displayed on three pages; the first was the consent form, and the others were 13- and 12-item questionnaires, respectively. The survey link was available from July 5, 2017, to September 18, 2017. A convenience sample of associations was based on their willingness and costs to deploy the survey.

The administrators of the Canadian Association of Emergency Physicians ( $\mathrm{n}=1,447)$, the European Association of Poison Centres and Clinical Toxicologists $(\mathrm{n}=291)$, the American Academy of Clinical Toxicologists $(n=504)$, the Asian Pacific Association of Medical Toxicologist $(n=344)$, the American College of Medical Toxicology $(n=680)$, and the Association des Pharmaciens d'Établissements de Santé of Quebec $(\mathrm{n}=1,978)$ sent a survey link to their membership. A single reminder was sent 2 weeks before closing the survey. Participation was voluntary. Due to known crossmemberships, eligible respondents were asked to complete the survey once only. Only physicians, pharmacists, or other clinicians managing alcohol withdrawal were eligible to participate. Informed consent was obtained at the beginning of the survey. Answers were anonymous. Ten minutes was the estimated time to complete the survey. This study was approved by our institutional Research and Ethics Board. The CHERRIES checklist was used to report the results. ${ }^{8}$

\section{Survey design}

The questionnaire's content was based on a literature review performed by local experts with backgrounds in clinical research and toxicology. The questionnaire 
was developed in English and French and was piloted among five local emergency physicians. Written feedback was requested and received by email. Respondents were asked to rank from 1 to 3, three literature-based definitions of resistant alcohol withdrawal, with choice 1 being their preference. Respondents were required to comment on whether they considered these definitions equivalent. Additionally, they could specify their own criteria for resistant alcohol withdrawal. Answers could be reviewed and changed using the "Back" button at any time before submitting their questionnaire. The survey questions are in supplementary material Appendix 1.

\section{Data analysis}

All answers were collected with the SimpleSurvey platform and extracted on an Excel spreadsheet (Microsoft Corp, Redmond, WA). An independent statistician eliminated duplicates by comparing IP addresses and demographics; only identical matches were deleted. We summarized categorical responses with counts and proportions. Median and interquartile ranges (IQRs) were calculated for the continuous responses. Benzodiazepine equivalence was defined as diazepam $5 \mathrm{mg}=$ lorazepam $1 \mathrm{mg}$ to express diazepam dosing equivalents (DEQ). ${ }^{9}$ Statistical analyses were performed with $\mathrm{R}$ studio version 1.1.447 (RStudio, Inc., Boston, MA). The response rate could not be accurately estimated because an unknown proportion of recipients received more than one invitation due to overlap in membership/mailing lists. We therefore defined the participation rate as the proportion of respondents who agreed to participate divided by the number of times the survey link was opened. Surveys that were not completed for at least one question were excluded.

\section{RESULTS}

\section{Respondent characteristics}

The response rate was $8.6 \%(453 / 5244)$, the participation rate was $48.5 \%$ (453/934), and the completion rate was $70.4 \%$ (319/453). Respondents totalling 384 provided adequate data for analysis; 302 (78.6\%) were attending physicians, 344 (89.6\%) had a full-time practice, and $270(70.3 \%)$ were practising emergency medicine. Most respondents (73.7\%) were practising for less than 20 years, and 223 respondents $(58.1 \%)$ treated

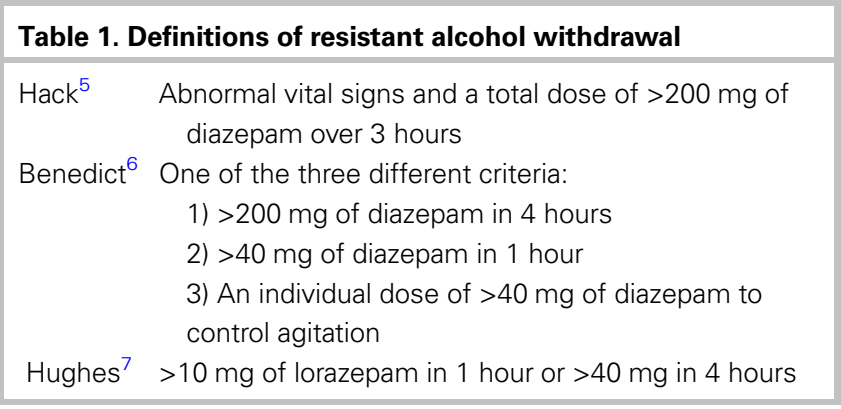

alcohol withdrawal patients at least once per week. Full respondent characteristics are shown in the supplementary material, Appendix Table 1.

\section{Resistant alcohol withdrawal definition}

Table 1 shows the literature-based definitions. Our survey used Benedict's definition as included in the research protocol available on www.clinicaltrial.gov, which was different in the final publications. ${ }^{10}$ We referred to it as the protocol number definition in the survey. Resistant alcohol withdrawal was described as a high benzodiazepine dosage (i.e., diazepam $40 \mathrm{mg}$ in 2 hours) by 244 respondents $(63.5 \%)$ followed by seizures by 98 respondents $(25.5 \%)$. These criteria were not mutually exclusive. Among the 146 respondents who quantified "high-dose benzodiazepine," the median dose was $40 \mathrm{mg}$ per hour (IQR; 20-50) of DEQ. See Table 2 for the full distribution of criteria surveyed.

The number of clinicians who ranked the three literature-based, resistant alcohol withdrawal definitions

\begin{tabular}{|c|c|}
\hline Criteria suggested* & Response rate $\mathrm{n}(\%)$ \\
\hline $\begin{array}{l}\text { High benzodiazepine dosage } \\
\text { (i.e., } 40 \mathrm{mg} \text { of diazepam over } 2 \text { hours) }\end{array}$ & $244(63.5)$ \\
\hline Seizures & $98(25.5)$ \\
\hline Persistent tachycardia & $62(16.1)$ \\
\hline High score on evaluation tool & $54(14.1)$ \\
\hline No definition ${ }^{\dagger}$ & $10(2.6)$ \\
\hline Not commonly seen in practice ${ }^{\ddagger}$ & $10(2.6)$ \\
\hline Agitation or confusion & $6(1.6)$ \\
\hline Other & 42 (10.9) \\
\hline
\end{tabular}


were Hack, 103 (26.8\%); Benedict, 108 (28.1\%); Hughes, 109 (28.4\%); and 65 (16.9\%) did not respond; $222(57.8 \%)$ respondents said these definitions were not equivalent.

\section{DISCUSSION}

Clinicians' individual definitions of resistant alcohol withdrawal are largely based on the total benzodiazepine dose required to control symptoms. The median hourly dose provided by respondents is consistent with the literature-based definitions. However, no single definition of resistant alcohol withdrawal was preferred, because answers were distributed equally over notably distinct definitions.

This survey raises questions whether variability among clinicians in diagnosing resistant alcohol withdrawal affects patient outcome. The IQR calculated for high benzodiazepine dose partially illustrates that variation. Our results begin a discussion to improve understanding this entity. Resistant alcohol withdrawal should be suspected when using more than $40 \mathrm{mg}$ of DEQ per hour and when the patient is worsening or not improving, despite closely repeated doses (seizures, persistent tachycardia, high score on assessment tool, and so on). This relatively new entity needs to be formally characterized in cohort studies to improve our understanding. Expert consensus could provide some definitional guidance to help future studies assess therapeutic interventions.

\section{Strengths and limitations}

Although we were unable to calculate cross-membership and ineligibility of individuals who received the link, we screened a large sample of clinicians using a rigourous method and provided a conservative participation rate. We pretested the questionnaire and allowed respondents to add information to their answers if desired. The dropout rate was $15.2 \%$. The same link included a second survey addressing different clinical questions about alcohol withdrawal, which will be discussed separately, and might have prolonged the survey duration contributing to dropout. Deployment during summer months in the Northern hemisphere could have limited the response rate. Multiple reminders could have improved the response rate.

Additionally, we surveyed members of clinical toxicology and emergency medicine associations, which may limit the generalization of our results. However, alcohol withdrawal is often diagnosed and treated in emergency departments with the assistance of clinical toxicologists. ${ }^{11,12}$ A selection bias may have been created by using a convenience sample of associations. Also, the term persistent tachycardia was not clearly defined, which may have led to wide interpretation. We were unable to find previous works that either discussed heart rate range for persistent tachycardia or other addressed clinician opinions on this topic.

\section{CONCLUSION}

Resistant alcohol withdrawal is an ill-defined clinical entity of unknown prevalence, defined by high-dose benzodiazepines (more than $40 \mathrm{mg}$ of DEQ per hour), seizures, and tachycardia. We hope our findings guide future consensus work towards a formalized definition that allows future standardized research to ultimately improve patient care in terms of a reduction in mortality, morbidity, and health system costs.

\section{SUPPLEMENTARY MATERIAL}

The supplementary material for this article can be found at https://doi.org/10.1017/cem.2019.421.

Competing interests: None declared.

\section{REFERENCES}

1. Long D, Long B, Koyfman A. The emergency medicine management of severe alcohol withdrawal. Am 7 Emerg Med 2017;35(7):1005-11.

2. Rehm J, Mathers C, Popova S, et al. Global burden of disease and injury and economic cost attributable to alcohol use and alcohol-use disorders. Lancet (London, England) 2009;373 (9682):2223-33.

3. Mayo-Smith MF. Pharmacological management of alcohol withdrawal. A meta-analysis and evidence-based practice guideline. American Society of Addiction Medicine Working Group on Pharmacological Management of Alcohol Withdrawal. 7AMA 1997;278(2):144-51.

4. Lejoyeux M, Solomon J, Ades J. Benzodiazepine treatment for alcohol-dependent patients. Alcobol Alcobol 1998;33(6):563-75.

5. Hack JB, Hoffmann RS, Nelson LS. Resistant alcohol withdrawal: does an unexpectedly large sedative requirement identify these patients early? $\mathcal{f}$ Med Toxicol 2006;2(2):55-60.

6. Hughes D. Benzodiazepine-refractory alcohol withdrawal. REBEL EM; 2016 [updated April 28, 2016]. Available at: 
http://rebelem.com/benzodiazepine-refractory-alcohol-withdrawal/ (accessed January 13, 2019).

7. Benedict NJ, Wong A, Cassidy E, et al. Predictors of resistant alcohol withdrawal (RAW): a retrospective case-control study. Drug Alcohol Depend 2018;192:303-8.

8. Eysenbach G. Improving the quality of Web surveys: the Checklist for Reporting Results of Internet E-Surveys (CHERRIES). 7 Med Internet Res 2004;6(3):e34.

9. Dundee JW, McGowan WAW, Lilburn JK, McKay AC, Hegarty JE. Comparison of the actions of diazepam and lorazepam. Br 7 Anaesth 1979;51:439-46.
10. US National Library of Medicine. ClinicalTrials.gov. Clinical quality improvement of benzodiazepine-resistant alcohol withdrawal syndrome (NCT01652326); 2012. Available at: https://clinicaltrials.gov/ct2/show/NCT01652326 (accessed October 4, 2017).

11. Hoskins R, Benger J. What is the burden of alcohol-related injuries in an inner city emergency department? Emerg Med $72013 ; 30(3): \mathrm{e} 21$.

12. Cherpitel CJ, Ye Y. Trends in alcohol- and drug-related emergency department and primary care visits: data from four U.S. national surveys (1995-2010). I Stud Alcohol Drugs 2012;73(3):454-8. 